\title{
ON THE GENUS OF CURVES OF AN ALGEBRAIC SYSTEM
}

\author{
BY \\ WEI-LIANG CHOW
}

Professor C. Chevalley has recently pointed out to me the desirability of having a simple algebraic proof of the following theorem: The genus of any curve of an algebraic system of algebraic curves is not greater than the genus of the generic curve of the system. The theorem seems to be well known, but I have sought in vain for a proof of it in the literature $\left({ }^{1}\right)$. We shall give here a purely algebraic proof of this theorem, based on the idea of the associated form of an algebraic variety, which was introduced by van der Waerden and myself some years ago $\left.{ }^{2}\right)$.

Let $\mathbb{S}$ be an irreducible algebraic curve of order $p$ in the projective space $\mathfrak{S}_{m}$ defined by the associated form

$$
F(C ; U, V)=\sum C_{i_{1}} \cdots i_{p}, i_{1} \cdots i_{p} U_{i_{1}} \cdots U_{i_{p}} V_{j_{1}} \cdots V_{i_{p}},
$$

where the $(C)$ are the coefficients, which can be taken as the coordinates of the curve $\mathfrak{E}$, and the $(U),(V)$ are indeterminates (they are, in fact, the coefficients of the generic hyperplanes $\sum_{i=0}^{m} U_{i} Y_{i}=0, \sum_{i=0}^{m} V_{i} Y_{i}=0$ in $\mathfrak{S}_{m}$ ). The curve $\mathbb{E}$ can also be defined by a set of equations

$$
f_{j}(B, Y)=0
$$

derived from the associated form (1) by rational process $\left({ }^{3}\right)$, so that the coefficients $(B)$ in $(2)$ are forms in $(C)$. It is essential to notice that the equations (2) remains valid for any value of $(C)$ corresponding to an associated form (1), so that in particular the relation between (1) and (2) is preserved under any specialization of $(C)$. The same remark applies to all succeeding steps of our argument. A necessary and sufficient condition for the genus $g(\mathfrak{E})$ of $\mathfrak{E}$ to be less than or equal to $g^{\prime}$ is that for every (or infinitely many) $n$ there exists a linear series of order not greater than $n$ and dimension not less than $n-g^{\prime}$. We shall show that this is an algebraic condition in terms of the $(C)$.

Consider the variety $\mathfrak{S}^{n}$ formed by the totality of all groups of $n$ points

Presented to the Society, June 19, 1948; received by the editors December 10, 1947.

(1) After the above note was written, it came to my attention that B. Segre, in a paper On limits of algebraic varieties, Proc. London Math. Soc. vol. 47 (1942) pp. 351-403, has also given a proof of this theorem. His proof, quite different from ours, is however not purely algebraic, as it uses the limit process.

(2) Chow and van der Waerden: Zur algebraischen Geometrie. IX. Ueber zugeordnete Formen und algebraische Systeme von algebraischen Mannigfaltigkeiten, Math. Ann. vol. 113 (1937) pp. 692-704.

(8) Chow and van der Waerden, loc. cit. p. 699, Satz 3. 
on $\mathfrak{E}$. By a group of $n$ points is meant a set of points on $\mathbb{E}$, to each of which is assigned a certain positive integer, called its multiplicity, such that the sum of all such multiplicities is equal to $n$. If we take as coordinates of such a group of $n$ points the coefficients $(P)$ of its associated form $P(V)$ $=\sum P_{j_{1}} \cdots j_{n} V_{j_{1}} \cdots V_{j_{n}}$, where the summation extends over all possible combinations (repetitions included) of the integers $0,1,2, \cdots, m$ in sets of $n$ elements, the variety $\mathbb{S}^{n}$ is then represented as an algebraic variety. In fact, the condition that $P(V)$ represents a group of $n$ points on $\mathbb{E}$ is expressed by the assertion that there exist $n$ solutions $\left({ }^{4}\right)\left(Y^{i}\right), i=1,2, \cdots, n$, of $(2)$ and a quantity $\lambda \neq 0$, such that the following equation

$$
P(V)=\sum P_{j_{1} \cdots j_{n}} V_{j_{1}} \cdots V_{i_{n}}=\lambda \sum_{i=1}^{n}\left(\sum_{j=0}^{m} Y_{j}^{i} V_{j}\right)
$$

holds identically in $(V)$. Equating the coefficients of the corresponding terms on both sides of this equation, we have

$$
P_{j_{1}} \cdots j_{n}=\lambda \sum Y_{j_{1}}^{1} \cdots Y_{j_{n}}^{n} \text {, }
$$

where the summation extends over all the permutations of the set $\left(j_{1}, \cdots, j_{n}\right)$. Since $\lambda \neq 0$, this is equivalent to a system of equations of the form

$$
P_{j_{1}} \cdots i_{n} \sum Y_{k_{1}}^{1} \cdots Y_{k_{n}}^{n}=P_{k_{1}} \cdots k_{n} \sum Y_{i_{1}}^{1} \cdots Y_{j_{n}}^{n} \cdot
$$

Eliminating the $\left(Y^{i}\right)$ between the equations (3) and the equations (2) for $\left(Y^{i}\right)$, that is, the equations $f_{j}\left(B, Y^{i}\right)=0$, we arrive at a set of equations in the (P)

$$
K_{j}^{n}(P)=0,
$$

which evidently define the variety $\mathfrak{S}^{n}$.

Let $\mathfrak{S}$ be a linear system of hypersurfaces of order $h$ in $\mathbb{S}_{m}$, with the property that no hypersurface of the system contains the curve $\mathbb{S}$ and not all hypersurfaces of the system contain any one fixed point of $\mathfrak{E}$. The system $\mathfrak{E}$ will cut out on the curve $(S$ a linear series of order $q=h p$, and we assume that $h$ is so large that $q>n$. Two point groups $P(V), Q(V)$ on $\mathbb{E}$, both of order $n$, are called quasi-equivalent with respect to the system $\mathfrak{E}$, if there exists a group of $q-n$ points on $\mathfrak{E}$, with the associated form $R(V)$, such that the point groups $R(V) P(V), R(V) Q(V)$ are two groups of the linear series cut out on $\mathfrak{C}$ by $\mathfrak{S}$. This condition of quasi-equivalence between the point groups $P(V)$ and $Q(V)$ (with respect to $\mathfrak{S}$ ) can also be expressed as an algebraic condition between the coordinates $(P),(Q)$ of the two corresponding points on the variety ${ }^{n}$. To show this, let $H(W, V)$ be the associated form of the

(4) Here as well as in the following, the trivial solution, consisting of all zeroes, is always excluded. 
linear series $\mathfrak{S}$, where the $(W)$ are the coefficients determining the individual groups of the series and the $(V)$ are as before the coefficients of a generic hyperplane in $\mathfrak{S}_{m}$. The condition can then be expressed thus: There exist $\left(W^{\prime}\right)$, $\left(W^{\prime \prime}\right), R(V)$ such that

$$
H\left(W^{\prime}, V\right)=\lambda R(V) P(V), \quad H\left(W^{\prime \prime}, V\right)=\mu R(V) Q(V) \quad(\lambda, \mu \neq 0) .
$$

One concludes just as before that this is equivalent to a system of equations of the form

$$
\begin{aligned}
& H_{j_{1} \cdots i_{q}}^{\prime} \sum P_{i_{1} \cdots i_{n}} R_{i_{n+1} \cdots i_{q}}=H_{i_{1} \cdots i_{q}}^{\prime} \sum P_{i_{1} \cdots j_{n}} R_{j_{n+1} \cdots j_{q}}, \\
& H_{i_{1} \cdots j_{q}}^{\prime \prime} \sum Q_{i_{1} \cdots i_{n}} R_{i_{n+1} \cdots i_{q}}=H_{i_{1} \cdots i_{q}}^{\prime \prime} \sum Q_{j_{1} \cdots j_{n}} R_{j_{n+1} \cdots j_{q}},
\end{aligned}
$$

where the $\left(H^{\prime}\right),\left(H^{\prime \prime}\right)$ are forms in $\left(W^{\prime}\right),\left(W^{\prime \prime}\right)$ respectively. Eliminating the $\left(W^{\prime}\right),\left(W^{\prime \prime}\right),(R)$ between the equations (5) and the equations $K_{j}^{q-n}(R)=0$, we arrive at a set of equations

$$
J_{j}(P, Q)=0,
$$

which is the algebraic condition for the quasi-equivalence with respect to $\mathfrak{S}$.

For each fixed point $P(V)$ of $\mathbb{S}^{n}$, the set of $Q(V)$ satisfying the equations (6) and the equations $K_{j}^{n}(Q)=0$ is evidently an algebraic variety, which is the

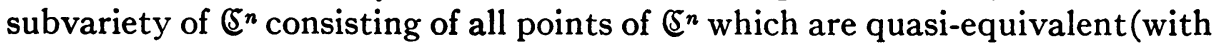
respect to $\mathfrak{S}$ ) with $P(V)$. The condition that this variety has a dimension not less than $n-g^{\prime}$ is expressed by eliminating the $(Q)$ between the equations (6), the equations $K_{j}^{n}(Q)=0$, and the $n-g^{\prime}$ generic linear equations $\sum_{j} U_{j}^{(i)} Q_{j}=0$ $\left(i=1, \cdots, n-g^{\prime}\right)$, where the $U_{j}^{(i)}$ are again indeterminates. The resultants of this elimination are forms in the indeterminates $U_{j}^{(i)}$. Setting each coefficient of these forms to zero, we arrive at a set of equations

$$
T_{j}(P)=0
$$

which is the condition that the set of points $Q(V)$ of $\sigma^{n}$ quasi-equivalent (with respect to $\mathfrak{S}$ ) with the point $P(V)$ forms a variety of dimension not less than $n-g^{\prime}$. Lastly, the condition that such a point $P(V)$ exists on $\mathfrak{S}^{n}$ is found by eliminating $(P)$ from (7) and (4), and we get a set of equations

$$
G_{j}(C)=0
$$

in the coefficients $(C)$ of the curve $\mathbb{E}$, which is necessary and sufficient for the existence of a point group of order $n$ on $\mathfrak{E}$, such that the set of all point groups of order $n$ on $(\mathbb{S}$ which are quasi-equivalent (with respect to $\mathfrak{E}$ ) with it forms a variety of dimension not less than $n-g^{\prime}$.

We shall now show that the existence of a "quasi-equivalent system" of order $n$ and dimension $r$ implies the existence of a linear series on $\mathbb{5}$ of order $n$ and dimension $r$. For two point groups $P(V), Q(V)$ are quasi-equivalent with respect to $\mathfrak{Q}$ if and only if there exist two integral divisors $\bar{H}^{\prime}, \bar{H}^{\prime \prime}$ of 
order at most equal to $q$ and containing only prime divisors corresponding to the multiple points of $\left(\mathcal{C}\right.$, such that the divisor $\bar{H}^{\prime} \bar{P}$ is equivalent to $\bar{H}^{\prime \prime} \bar{Q}$, where $\bar{P}, \bar{Q}$ now stand for any (of the finite number of possible) divisors corresponding to the point groups $P(V), Q(V)$ respectively. This means that every $\bar{Q}$ is equivalent to a divisor $\left(\bar{H}^{\prime} / \bar{H}^{\prime \prime}\right) \bar{P}$ of order $n$. Since there are only a finite number of such divisors $\bar{H}^{\prime} / \bar{H}^{\prime \prime}$, we conclude that the entire "quasiequivalent system" determined by $P(V)$ dissolves into a finite number of equivalent systems of divisors or linear series of order $n$, at least one of which must of course have the same dimension $r$. Hence our assertion is proved.

Consider now an irreducible algebraic correspondence in which to each point $X$ of a variety $\mathfrak{X}$ there corresponds an irreducible curve $\mathfrak{E}_{x}$ in a variety $\mathfrak{V}$ in $S_{m}$. Let $F\left(C_{x} ; U, V\right)$ be the associated form of $\mathfrak{C}_{x}$; we have then a correspondence between the point $X$ and the coordinates $\left(C_{x}\right)$ of the associated form. Let $\left(C_{a}\right)$ be a specialization of $\left(C_{x}\right)$ corresponding to the specialization $X \rightarrow a$; then the form $F\left(C_{a} ; U, V\right)$ is the associated form of a curve $\mathfrak{G}_{a}$ in $\mathfrak{Y}$. We assume that the curve $\mathfrak{C}_{a}$ is irreducible. In applying the above considerations to the curve $\mathfrak{E}_{x}$, we take for $\mathfrak{S}$ the linear system of all hypersurfaces of order $h$ in $\mathfrak{S}_{m}$ which do not contain the curve $\mathfrak{S}_{a}$ and hence also do not contain $\mathfrak{E}_{x}$. For sufficiently high $h$ the linear system $\mathfrak{S}$ contains a subsystem which cuts out on $\mathfrak{E}_{x}$ a complete series of order $n$, which must have a dimension not less than $n-g\left(\mathbb{S}_{x}\right)$. Then the "quasi-equivalent system" determined by any point group (corresponding to any integral divisor) of the complete series must obviously have at least this same dimension. Hence the $\left(C_{x}\right)$ satisfy the equation (8) with $g^{\prime}=g\left(\mathbb{S}_{x}\right)$; and consequently the $\left(C_{a}\right)$ satisfy also the equations (8), also with $g^{\prime}=g\left(\mathbb{E}_{x}\right)$, and hence $g\left(\mathbb{E}_{a}\right) \leqq g\left(\mathbb{E}_{x}\right)$.

To complete the proof we must show that the so chosen linear system $\mathfrak{S}$, for sufficiently high $h$, contains a subsystem which cuts out on $\mathfrak{F}_{x}$ a complete series of order $n$. This follows from the fact that already the subsystem of $\mathfrak{W}$ consisting of all the hypercones passing through a generic $\mathfrak{S}_{m-3}$ has this property. This can be seen most easily by projecting the curves $\mathfrak{E}_{x}$ and $\mathfrak{E}_{a}$ from the generic $\mathfrak{S}_{m-3}$ into plane curves $\mathfrak{S}_{x}^{\prime}$ and $\mathfrak{S}_{a}^{\prime}$ in a generic plane; the system of hypercones passing through $\mathfrak{S}_{m-3}$ will then be projected into the system of all curves of order $h$ not containing the curve $\mathfrak{S}_{a}^{\prime}$ (and hence not containing the curve $\left(\mathfrak{F}_{x}^{\prime}\right)$ in the plane. Now the dimension of such a system of curves depends only on the order $h$ and the order of the curve $\mathfrak{S}_{a}^{\prime}$, and since $\mathfrak{F}_{x}^{\prime}$ and $\mathfrak{C}_{a}^{\prime}$ certainly have the same order, it follows that this system must also consist of all the curves of order $h$ not containing $\mathfrak{E}_{x}^{\prime}$. That this system of curves, for sufficiently high $h$, contains a subsystem which cuts out on $\mathfrak{C}_{x}^{\prime}$ a complete series of order $n$ is quite obvious. Thus everything is proved.

The Institute for Advanced Study, Princeton, N. J. 